\title{
The Business Transformation and Enterprise Architecture Framework The Applied Holistic Mathematical Model's Persistence Concept (AHMMPC)
}

\author{
ANTOINE TRAD \\ Institute of Business and Information Systems Transformation Management \\ Paris, FRANCE
}

\begin{abstract}
This article proposes a cross domain Applied Holistic Mathematical Model (AHMM) that is the result of a lifetime long research on various types of transformations, applied mathematics, software modelling, heuristic brain-like algorithms, business engineering, financial analysis and global system architecture. This ultimate research is based on an authentic and proprietary mixed research method that is supported by an underlining mainly qualitative holistic neural networks algorithms [1]. The proposed HMM formalism attempts to mimic and simulate some functions of the human brain, which uses empirical processes that are mainly based on the beam-search, like heuristic decision-making process that uses a persistence concept.
\end{abstract}

Key-Words: - Enterprise Architecture, Business Transformation Project, Business Transformation Manager, Integration Concept, Business Engineering, Applied Mathematical Models, Neural Networks, Holisms, Risk Management, Decision Making Systems and Knowledge Management Systems.

Received: July 14, 2020. Revised: October 23, 2020. Accepted: November 23, 2020. Published: December 10, 2020.

\section{Introduction}

This article proposes a persistence concept for the cross-business domain mathematical model that has the acronym, the Applied Holistic Mathematical Model Persistence Concept (AHMMPC). The underlying mathematical model, is the result of a lifetime long research on business/technical transformations, applied mathematics, software modelling, artificial intelligence, business engineering, financial analysis and global enterprise architecture. The proposed concept is based on an authentic and proprietary, the mixed research method that is supported by mainly qualitative holistic reasoning model [1][2]. The proposed AHMMPC formalism attempts to mimic some functions of the human brain, which uses empirical processes that are mainly based on the beam-search, like heuristic decision-making processes that are synchronized with a persistence subsystem.

\section{Problem Formulation}

The AHMMPC can be used to implement a decision making system or an expert system that can integrate in the enterprise's business, information and communication technology environments. The
AHMMPC uses a behaviour driven development environment or a natural language environment that can be easily adopted by the project's development teams [3][4]. The AHMMPC offers a high level implementation environment that can be used by any team member without any prior computer sciences qualification. The AHMMPC can be used also to model Enterprise Architecture (EA) blueprints, business transformation projects or knowledge management systems; it is supported by many real-life cases of various business domains. The uniqueness of this research is that the AHMMPC promotes a holistic unbundling process, the alignment of various EA standards and transformation strategies to support business transformation projects and a persistence concept [5].

\subsection{Supporting persistence}

Actual archaic Business Transformation Projects and enterprise architecture projects (or simply the Project) are managed as separate isles where their internal and external components are archaic part of the enterprise's Information and Communication System (ICS). As already mentioned, the AHMMPC is based on many real-life cases and uses a model that can be used in a large variety of applications' persistence concepts, like: 1) business transformation projects' global persistence concept; 
2) business engineering projects' global persistence concept; 3) critical success factors management; and 4) EA global persistence procedures. This article recommends that the ICS's Decision Making System (DMS) uses the AHMMPC instance to solve persistence problems by offering a set of patterns or blueprints, for any type of persistence activity to support central qualitative method based on a beam search (heuristic tree) that uses quantitative methods at its nodes. The proposed AHMMPC's implementation is complex and needs a profound understanding of many data manipulation and design fields. The DMS' actions produce solutions, which have the form of technical and managerial recommendations, can be applied by the business environment's architects, business managers, business analysts and project engineers to enforce the implementation of the transformation processes and their underlying persistence concept. A DMS is a multi-objective, multi-project, multi-factor (CSF) and Project problem in the context of a complex implementation phase. The DMS attempts simultaneously to maximize the success rate. Such processes should surpass the business environment's currently used usual DMs. The AHMMPC is a model first modelling environment that is supported by an applicable framework [6][7].

\subsection{The background}

This article's background combines Knowledge Management (KM), innovative decision making systems approach, enterprise architecture, heuristics/mathematical models, information technology management, business transformation initiatives and business engineering fields [8]. The major strategic technology trend is artificial intelligence based systems; so the author concludes that building an innovative AHMMPC model. The AHMMPC model enables the implementation of a generic and cross-functional reasoning engine which manages sets of factors which can be applied to a Project. This article's author based his research model mainly on intelligent neural networks' related persistence which can execute specific calls to quantitative modules and is supported by information technology driven development models, where both disciplines, applied mathematics and information technology models are complementary, due to the use of many existing industry standards, like for example the Architecture Development Method (ADM) [9]. The author developed a genuine research framework that is founded on DMS microartefacts that in turn are based on existing standards. The Business Transformation Manager (or simply the Manager) or an enterprise architect can integrate an AHMMPC based Project in the company's architecture roadmap, to support its complex data access scenarios. The AHMMPC model aim is to deliver a generic persistence pattern that is capable to deliver just-in-time solutions in the form of applicable actions for data manipulation. To achieve this goal, the research methodology is based on: 1) a multi-domain literature review; 2) a qualitative methodology; 3) a quantitative methodology; and 4) a persistence engineering oriented proof of concept (or a controlled experiment); which is the optimal methodology applied in information technology, applied mathematics and other types of engineering projects [10]. In general, Projects lack holistic and synchronized persistence implementation concepts An adapted persistence development and iterative process of operations can be adapted to support synchronized the implementation approach. The AHMMPC classifies CSFs in Critical Success Areas (CSA) groups. In Projects, there is pressure to offer robust systems. The mentioned pressure is the main cause that a Project can fail or is simply cancelled [11].

\section{The Research Process}

\subsection{The Research's Uniqueness}

The uniqueness of this research is that the AHMMPC promotes a holistic persistence process, the alignment of various EA standards and data management strategies to support Projects. The uniqueness of this research project is based on its holistic approach that:

- Intersects: 1) Project; 2) AHMMPC; 3) software modelling and architecture; 4) business engineering; 5) financial analysis; 6) data persistence; and 6) global enterprise architecture. And offers a methodology and framework.

- Using a scholar search engine (like Google's) combining the previous topics, clearly shows the uniqueness and the lead of the author's works.

This research question and its analysis are genuine and intended to close the immense gap in this field.

\subsection{The Research Gap}

The AHMMPC gap is analyzed by using a central qualitative engine, which is based on beam-search heuristics [12]. To close or at least narrow the gap in the mentioned research field, the author proposes a holistic approach that unifies the following:

- An applied mathematical model.

- A concrete AHMMPC.

- The CSA and CSF management. 
- Implementation strategy for the development of persistence microartefacts.

\subsection{The Research Framework}

AHMMPC based DMS uses CSFs, which are managed by this research's framework. The AHMMPC based framework supports a complex formalism that combines persistence mechanisms with EA, DMS and various standards with a mathematical model. Unfortunately, most of the existing formalisms are archaic, and silo-built. The AHMMPC is a part of the Decision making module $(\mathrm{Dm})$ and the Architecture module $(\mathrm{Am})$, that in turn are parts of the framework. In this article, the author proposes a set of AHMMPC managerial and technical recommendations on how to used a framework in a Project. The AHMMPC supports standards, like The Open Group's Architecture Framework's (TOGAF) ADM, where each Project microartefact circulates through its phases. In this research, TOGAF is used, but other architecture frameworks can also be used. These microartefacts contain their private sets of CSFs that can be applied [13]. The actual research article and the resultant experiment are also a part of the Selection management, Architecture-modelling, Controlmonitoring, Decision making, Training management, Project management, Finance management, Geopolitical management, Knowledge management and Implementation management Framework (SmAmCmDmTmPmFmGmKmImF, for simplification reasons, in further text the term the Framework will be used). The Framework is not a black-box product to be applied as-is, it is rather a transformation strategy that offers recommendations and vision on how to implement a Project.

This article can be considered as a non-conventional and evolutionary one, in the field of holistic Projects and EA projects and their underlying mechanisms. This article's research question is: "Can an applied holistic mathematical model be used to implement business transformation projects and enterprise architecture projects underlying persistence mechanisms?". It was formulated after an extensive literature review.

\subsection{The Research's Literature Review}

As already mentioned, this research cluster that is focused on Projects and EA; and owns an extensive literature library that contains major publications related to the research topics. For this research article, the literature review process has focused on the following subjects:

- Modelling persistency with a mathematical model.

- The role of CSFs in a mathematical model.
- Model driven architecture: is defined by the Object Management Group.

- A decision making system's persistency.

- A mathematical model to support persistence.

- Multi-criteria or a multi-factor model for decision making needs a mixed method based on qualitative and quantitative criteria

\subsection{Empirical Engineering Research Model}

This research article is based on an empirical engineering research approach because of the following facts [10]:

- It uses an authentic mixed method.

- Today we have five classes of research methods.

- Engineering and applied mathematics researchers are very poor at making theories and related research explicit.

- This research uses Action Research (AR) which helps the researchers to solve a real Project problem [15].

- A controlled experiment or a Proof of Concept $(\mathrm{PoC})$ is a software prototype of a testable hypothesis where one or more CSFs.

This research article is an empirical research and it includes a proof of concept [10]; and proposes an AHMMPC based on a DMS that uses sets of CSFs:

- The mathematical model with its pool of microartefacts.

- The mathematical model's integration in the information and communication technology system; and especially its persistence mechanisms.

- The holistic management of the decision making system, using a mathematical model and persistence management subsystem.

\section{The Applied Mathematical Model}

The unbundling process insures that the ICS microartefacts are ready to be used; this is the most delicate and complex transformation undertaking in Projects and the main cause of their failure. The ICS unbundling microartefacts:

- Services unbundling procedures.

- Microartefacts Project development cycles.

- Transformation of software systems.

This research offers an instance of the mathematical model, the AHMMPC that is an abstract model containing a Mathematical Language (ML) that can be used to describe and implement the behavior of 
any business system and its persistence subsystem. The AHMMPC that is based on related research by many works, where managers, engineers, computer scientists, and economists can use AHMMPC to manage data access.

\subsection{A holistic approach}

A set of constraints exist that can make persistence subsystems difficult to implement. These constraints depend on the level of enterprise's global status and on the way Project development planning is managed. To understand better the notions of holism and the underlying mathematical models, the evolution of technology is important, in order to show that this integration is possible.

\subsection{A mathematical model's basics}

Polderman and Willems [15] argue that mathematical model is a subset of real world's realities and that the mathematical model is a description of such a reality based on a dynamical system as subset of time-evolution that can be traced with timestamps. A mathematical model contains interrelated equations; or diagrams. In the AHMMPC the reality is the business context that understands: 1) enterprise architecture; 2) business transformations; 3) business and software engineering; 4) algorithmics; and 5) data persistence. There is a need for holistic perspective for the Project and its underlying mathematical model structure to prove if it can succeed [16].

\subsection{The structure}

The structure is used to resolve interdependencies Project resources. These resources interdependencies can result in many silo subprojects. The use of a AHMMPC can insure a successful outcome. A mathematical model's structure represents the mapping relations between Project's resources to be persisted.

Critical Success Areas (CSA), Factors (CSF) are the kernel elements of decision Making. The AHMMPC is based on CSA which are categories of sets of CSF where in turn, each CSF is a set of selected Key Performance Indicators (KPI), where: 1) each CSA corresponds to a Project domain, like for example, persistence mechanisms.

\subsection{Unit of Work}

A holistic alignment and classification of all the Project's resources must be done, so that the unbundling process can start. A holistic alignment needs also to define the AHMMPC Unit of Work. The mapping concept supports the interoperability between all the Project's modules and enables the use of persistence microartefacts that include the needed access mechanisms.

\subsection{Microartefacts}

A persistence microartefact is any data management microartefact that is a part of the AHMMPC and which interacts with a multitude of data media. A persistence microartefact uses the ADM to assist the Project's implementation process [9]. The AHMMPC includes various types of data access mechanisms that to make the data integration flexible and to avoid archaic data accesses.

\subsection{Nomenclature of the model}

$\begin{array}{ll}\text { mcEnterprise } & \text { A micro enterprise component } \\ \text { mcRequirement } & \text { A micro requirement } \\ \text { mcArtefact } & \text { A microartefact } \\ \text { action (or action) } & \text { An atomic service execution scheme } \\ \text { mcIntelligenceArtefact } & \text { A set that contains: dynamic basic intelligence + governance + persistence + } \\ & \text { traceability + data_xsd + resources } \\ \text { mcArtefactDecisionMaking } & \text { A microartefact_decision making entity } \\ \text { mcArtefactScenario } & \text { A microartefact scenario }\end{array}$

Figure 1. The nomenclature.

The AHMM, is based on a light version of the ADM and is a part of the Framework that uses services architecture. Services architecture enables unprecedented agility, business benefits and infrastructure scalability. These facts can be formalized with a mathematical model that needs a nomenclature, as shown in Fig. 1.

\begin{tabular}{|c|c|}
\hline microRequirement & $=\mathrm{KPI}$ \\
\hline CSF & $=\sum \mathrm{KPI}$ \\
\hline CSA & $=\sum \mathrm{CSF}$ \\
\hline Requirement & $=\underline{\bigcup}$ microRequirement \\
\hline microKnowledgeArtefact & $=\underline{\bigcup}$ knowledgeItem $(\mathrm{s})$ \\
\hline (e)neuron & $=$ action + microKnowledgeArtefact \\
\hline microArtefact & $=\underline{U}(\mathrm{e})$ neurons \\
\hline microEnterprise & $=\underline{\bigcup}$ microArtefact \\
\hline (e)Enterprise & $=\underline{\bigcup}$ microEnterprise \\
\hline microArtefactScenario & $=\underline{\bigcup}$ microArtefactDecisionMaking \\
\hline IntelligenceComponent & $=\underline{U}$ microArtefactScenario \\
\hline OrganisationalIntelligence & $=\underline{\bigcup}$ InteligenceComponent \\
\hline
\end{tabular}

Figure 2. The basic model.

As shown in Fig. 2, the model has a composite structure that can be viewed as follows:

- The static view.

- The behavioral view.

- The skeleton of the Framework.

This research is a pioneering research work and is cross-functional and links all the Project's microartefacts to Projects [17].

AHMM $=\mathrm{ADM}+$ Organisationallntelligence $\quad(12)$

Figure 3. The applied mathematical Model. 
Actions research can be considered as a set of continuous beam-search heuristics processing phases and is similar to design and architecture processes, like the ADM [18]. The AHMMPC is an instance of the AHMM and is responsible for the qualitative heuristic process for Project's problem solving and synchronizes a set of ADMs, as shown in Figure 3.

\subsection{The Model's Critical Success Factors}

Based on the research process, the most important mathematical model's CSFs that are used are evaluated to the following:

\begin{tabular}{|l|l|l|l}
\hline Critical Success Factors & \multicolumn{2}{l|}{ KPIs } & Weightings \\
\hline CSF_AHMM_HolisicmEvolution & DifficultButImplementable - & From 1 to 10.6 Selected \\
\hline CSF_AHMM_Structure & Applicable & Applicable & From 1 to 10.10 Selected \\
\hline CSF_AHMM_CSA & Applicable & From 1 to 10.10 Selected \\
\hline CSF_AHMM_Microartefacts & - & From 1 to 10.10 Selected \\
\hline CSF_AHMM_Architecture & DifficultButImplementable - & From 1 to 10.7 Selected \\
\hline CSF_AHMM_TransformationaliModel & DifficultButImplementable _ & From 1 to 10.7 Selected \\
\hline CSF_AHMM_FunctionalLanguage & Applicable & From 1 to 10.10 Selected \\
\hline
\end{tabular}

Table 1. The critical success factors that have an average of 8.15 .

As shown in Table 1, the result's aim is to prove or justify that it is complex but possible to implement a mathematical model in the information system. The next CSA to be analyzed is the holistic management of the decision making system.

\section{The Information Communication System}

and

Today many technology (including persistence mechanisms) standards exist and their related tooling and development environments are supposed to support the iterative unbundling process of a traditional business and its information technology environments, through the execution of an agile process [19].

\subsection{Development/Operations Choreography}

and

Architecture, modelling, development, operations, integration and transformation tools/environments for persistence mechanisms are skeletons that should enclose various automated specialized microartefacts capabilities. The Framework offers the ML environment, which includes the formalism that can be used to enable fast persistence operations for development, operations, integration and testing iterations and to support data implementation processes. The AHMMPC formalism is based on existing proven standard data models and controls that are mainly based on service oriented architecture. The design first approach, is recommended to implement microartefacts. The AHMMPC formalism expresses a holistic structural concept or schema for the Project's global data management capabilities.

\subsection{Microartefacts deployment and agility}

Persistence microartefacts' manipulation is in fact, a set of micro-actions that manage various data access activities. The AHMMPC structure is used to generate microartefact instances and receives and evaluates data requests. In order to transform an existing enterprise data management system, an adapted AHMMPC formalism is needed to generalize the persistence concept.

\subsection{Architecture and development cycles}

AHMMPC needs a real world, technically agnostic, data management tool like the enterprise's Rapid Application Development (RAD) environments; where RAD tools have been the ICS's obsession since the right beginning of programming techniques [20]. RAD tools complicate the adoption of a holistic architectures. The integration of AHMMPC in the ICS' data management, is the backbone of the future n-tiered decoupled system. An adaptable cross-functional AHMMPC formalism is important for the future of any data management system and a holistic integration strategy has to be defined using a standardized methodology like TOGAF and its Archimate modelling environment.

\subsection{Architecture development method and} tests

The AHMMPC integration with the ADM, enables the automation and the auto-generation of the project's data management microartefacts. These microartefacts management scripts, circulate throughout all the ADM including tests. The major problem that causes a Project to be stopped or to fail, is the performance problem that in general in business enterprises is translated and justified by the human behavioral aspects.

\subsection{The Information and Communication Technology's Critical Success Factors}

Based on the research process, the most important ICS CSFs that are used are evaluated to the following: 


\begin{tabular}{|c|c|c|c|}
\hline Critical Success Factors & KPIs & & Weightings \\
\hline CSF_ICS_Development\&Tests & Immature & $\nabla$ & From 1 to 10.2 Selected \\
\hline CSF_ICS_DesignFirst & TooComplex & $\checkmark$ & From 1 to 10.6 Selected \\
\hline CSF_ICS_RAD4Persistence & Ready & $\nabla$ & From 1 to 10.9 Selected \\
\hline CSF__CS_Arch & Applicable & $\nabla$ & From 1 to 10.9 Selected \\
\hline CSF_ICS_ADM & Applicable & - & From 1 to 10.9 Selected \\
\hline CSF_ICS_PersistenceAdmin & TooComplex & - & From 1 to 10.6 Selected \\
\hline CSF_ICS_Tests & Applicable & - & From 1 to 10.9 Selected \\
\hline
\end{tabular}

Table 2. The critical success factors that have an average of 7.15 .

As shown in Table 2, the result tries to prove or justify that it is complex but possible to implement a mathematical model in the information and communication system. The next CSA to be analyzed is the holistic management of intelligent systems.

\section{The Decision Making and Knowledge Systems}

\subsection{Intelligent systems}

Intelligent systems management can be adapted to various types of Project's problems and information requests. The data/information requests are processed by using the AHMMPC that in turn is based on the selected CSAs and CSFs that are used in the DMS and the Knowledge Management System (KMS) which has a very complex system evolution nature as shown in Fig. 4 [21].

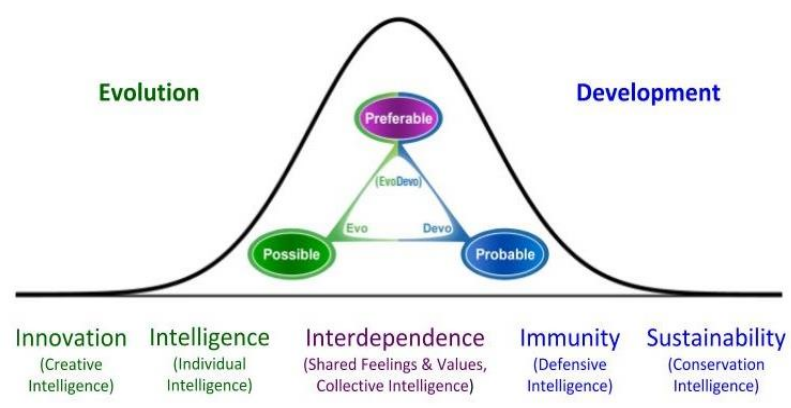

Figure 4. Complex system's nature and approach [21].

\subsection{Knowledge management system}

The proposed Framework relates and assembles all the Project's microartefacts and data resources; it links them also to the KMS and automates the autonomic data management microartefacts' instances. The AHMMPC system has to identify the initial set of CSFs to be used in the KMS and DMS.

\subsection{Decision making system}

The AHMMPC interfaces the
DMS, where any Project user can configure the types of microartefacts and CSFs to be used; these microartefacts use the AHMMPC choreography engine to manage persistence. The DMS' actions map to the various data management mechanisms to deliver information. The AHMMPC formalism is implemented in all of the Project's processes and the implementation of microartefacts to deliver data to the DMS; such a set of actions can be modelled and managed by the AHMMPC that is implemented with an experiment or a proof of concept.

\subsection{Intelligent systems' critical success factors}

Based on the research process, the most important intelligent system's CSFs that are used are evaluated to the following:

\begin{tabular}{|c|c|c|c|}
\hline Critical Success Factors & AHMM enhances: KPIs & & Weightings \\
\hline CSE_DMS_IntelligentSystems & Support & 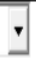 & From 1 to 10.9 Selected \\
\hline CSE_DMS_KMS_ Integration & Enables & 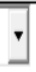 & From 1 to 10.10 Selected \\
\hline CSE_DMS DMP & Intgerates & I & From 1 to 10.10 Selected \\
\hline CSE_DMS_DMS_PersistenceN_anagement & Intgerates & \pm & From 1 to 10.10 Selected \\
\hline
\end{tabular}

Table 3. The critical success factors that have an average of 9.75 .

As shown in Table 3, the result tries to prove or justify that it is complex but possible to implement a decision making system in the information system.

\section{The Research Implementation}

\subsection{The research case}

This PoC's implementation uses the default demo application named Handle Claim Process case study that comes with the Archi tool, as the experiment's business case. The demo application is an insurance claims management system that has a client relations management system, claim files service, and customer file service. The demo application manages, registers, accepts, valuates and invoices claims activities. The demo application uses the Archi Archimate modelling tool for the proof of concept [22].

\subsection{The research proof of concept}

The AHMMPC research experiment or PoC was implemented using the research's cluster known as the Framework that had been developed by the author, using the Microsoft Visual Studio .NET, $\mathrm{C} / \mathrm{C}++$ and Java. The $\mathrm{PoC}$ is based on the AHMMPC and an internal set of CSFs' that are presented in Tables 1 to 3 . These CSFs have bindings to specific research resources, where the 
AHMMPC formalism was designed using microartefacts, object oriented and enterprise architecture methodologies and tools. The AHMMPC represents the relationships between this research's requirements, microartefacts (or building blocks), unique identifiers and the three defined CSAs.

Once the development setup interface is activated the natural language interface can be launched to implement the needed microartefact scripts to process the defined three CSAs. These scripts make up the kernel knowledge system and the AHMMPC set of actions that are processed in the background. The AHMMPC uses a database that automatically generates data management actions. This research's instance of the AHMMPC and its related CSFs were selected as demonstrated previously.

This article's three tables and the resultant Table 4, shows the AHMMPC is a dependent component and is bonded to the Framework's architecture, hence has to have a holistic persistence approach.

\begin{tabular}{|c|l|c|}
\hline CSA Category of CSFs/KPIs & \multicolumn{1}{|c|}{$\begin{array}{c}\text { Influences data } \\
\text { management/Persistence }\end{array}$} & $\begin{array}{c}\text { Average } \\
\text { Result }\end{array}$ \\
\hline $\begin{array}{c}\text { The Information and communication } \\
\text { technology yystem }\end{array}$ & Built on silos difficicult to transform & $\begin{array}{c}\text { From 1 to 10 } \\
7.15\end{array}$ \\
\hline The mathematical model's integration & Applicable & $\begin{array}{c}\text { From 1 to 10 } \\
8.15\end{array}$ \\
\hline The intelligent subsystems interfacing & Implementable & $\begin{array}{c}\text { From 1 to 10 } \\
\end{array}$ \\
\hline
\end{tabular}

Table 4. The data management/persistence research's outcome.

The Framework and hence the AHMMPC's main constraint is that CSAs for simple research projects, having an average result below 8.5 are failures. In the case of the AHMMPC's complex implementation, the average is 6.5 , because of the complexity, will be ignored. As shown in Table 4, this fact keeps the CSAs (marked in green) supports the conclusion that it is feasible. It means that such an AHMMPC formalism global integration will major face difficulties but possible.

\section{Conclusion and Recommendations}

Table 4 shows that AHMMPC implementation is not a very risky and is possible; this research work and its $\mathrm{PoC}$ have fully achieved the defined objectives. The resultant recommendations are:

- architecture methodologies improve the persistence concept.

- Unbundling the enterprise system to deliver the needed persistence microartefacts library.
- Develop an ICS based on the AHMMPC.

As shown in Figure 5, the AHMMPC instance is in all of the Project's processes; such a set of CSF mapped actions; like the ones presented in this chapter's experiment or a proof of concept.

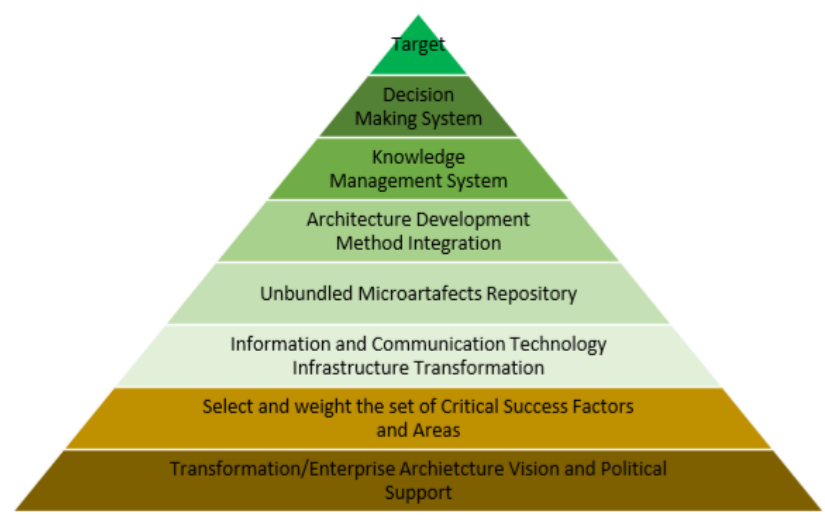

Figure 5. The proposed methodology and framework pyramid.

The AHMMPC is a part and is the skeleton of the Framework that uses microartefacts' scenarios to support just-in-time and system's requests. An instance of the AHMMPC is created at the Project's initialization phase and takes care of the data management subsystems. The aggregations of all the Project's CSA/CSF tables exceeds the defined minimum, the Project continues to its $\mathrm{PoC}$ or can be used for data management/persistence operations using the punctual calls to the Framework's methods.

This research phase is part of a series of publications related to Projects, decision making systems and enterprise architectures. This research is based on mixed action research model; where critical success factors and areas are offered to help Project architects to diminish the chances of failure when building data management/persistence subsystems. In this article, the focus is on the AHMMPC's formalism that defines a structured inter-relationship of data management/persistence microartefacts. AHMMPC is an important factor for the ICS's evolution. The most important managerial recommendation that was generated by the previous research phases was that the business transformation manager must be an architect of adaptive business systems.

To avoid costly scenario, the author recommends performing the Project operations through multiple independent sub-projects, where the priority is to transform the information system, structure a 
mathematical model, decision making system and global architecture.

\section{Future Research Directions}

The Framework future research efforts will focus on the holistic integration of the article's mentioned various fields, like economic intelligence, to increase success of transformational initiatives in a cross-functional environment.

\section{References:}

[1] Trad, A., Kalpić, D. The Business Transformation Framework and Enterprise Architecture Framework for Managers in Business Innovation-An applied holistic mathematical model (AHMM), IGI Journal of Science and Technology, 2019.

[2] Trad, A. An Applied Mathematical Model for Business Transformation and Enterprise Architecture-The Holistic Mathematical Model Integration (HMMI). IGI Book, 2019.

[3] Myers, B., Pane, J., Ko, A. Natural programming languages and environments. ACM New York, NY, USA. 2004.

[4] Della Croce, F., T'kindt, V. A Recovering Beam Search algorithm for the one-machine dynamic total completion time scheduling problem, Journal of the Operational Research Society, 53:11, 1275-1280. Taylor \& Francis. 2002.

[5] Farhoomand, A. Managing (e)business transformation. Palgrave. UK. 2004.

[6] IBM. Database Design Models - the UML Profile for Database Design. IBM. USA. 2001.

[7] Trad, A. The Transformation Framework Proof of Concept. Internal project and paper. IBISTM. France. 2019.

[8] Goikoetxea, A. A mathematical framework for enterprise architecture representation and design. International Journal of Information Technology \& Decision Making. Vol. 03, No. 01, pp. 5-32 (2004). USA. 2004.

[9] The Open Group. TOGAF 9.1. The Open Group. USA. Reviewed in August 2018, http://www.opengroup.org/subjectareas/enterpr ise/togaf . The Open Group. The Open Group. USA. 2011.

[10] Easterbrook, S., Singer, J., Storey, M., Damian, D. Guide to Advanced Empirical Software Engineering-Selecting Empirical Methods for Software Engineering Research. F. Shull et al. (eds.). Springer. 2008.

[11] Kornilova, I. DevOps is a culture, not a role! Meduim. 2017.

[12] Kim, K. \& Kim, Ki. Routing straddle carriers for the loading operation of containers using a beam search algorithm. Elsevier. Computers \& Industrial Engineering. Volume 36, Issue 1, 1999, Pages 109-136. 1999.

[13] Peterson, S. Why it Worked: Critical Success Factors of a Financial Reform Project in Africa. Faculty Research Working Paper Series. Harvard Kennedy School. 2011.

[14] Berger, J., Rose, J. Nine Challenges for eGovernment Action Researchers. International Journal of Electronic Government Research. 2015.

[15] Polderman, J., Willems, J. Introduction to Mathematical Systems Theory: A Behavioral Approach. (Texts in Applied Mathematics) 2nd Edition. Springer Verlag. Germany. 1998.

[16] Cardona, O. The Need for Rethinking the Concepts of Vulnerability and Risk from a Holistic Perspective: A Necessary Review and Criticism for Effective Risk Management. Taylor \& Francis Group. 2004.

[17] Agievich, V. Mathematical model and multicriteria analysis of designing large-scale enterprise roadmap. $\mathrm{PhD}$ thesis on the specialty 05.13.18 - Mathematical modelling, numerical methods and complexes of programs. 2014.

[18] Järvinen, P. Action Research is Similar to Design Science. Quality \& Quantity. February 2007, Volume 41, Issue 1, pp 37-54. Springer Verlag. Germany. Retrieved August 10, 2018, from

https://link.springer.com/article/10.1007/s1113 5-005-5427-1. 2007.

[19] Tidd, J., Bessant, J. Managing Innovation: Integrating Technological, Market and Organizational Change, Sixth Edition. New York: Wiley. USA. 2018.

[20] Kraisig, A., Rosélia, A., Welter, F., Haugg, I., Cargnin, R., Roos-Frantz, F., Sawicki, S., Frantz, Mathematical Model for Simulating an Application Integration Solution in the Academic Context of Unijuí University. December 2016. Procedia Computer Science 100:407-413. ScienceDirect. Elsevier. 2016.

[21] Foresight Guide. Five Goals of Complex Systems - Key Purposes and Processes of Life and Other Complex Adaptive Evo Devo Systems. Foresight University. CA, USA. 2017.

[22] Beauvoir, Ph., Sarrodie, J.B. Archi-The Free Archimate Modelling Tool. User Guide. The Open Group. UK. 2018.

\section{Creative Commons Attribution License 4.0 (Attribution 4.0 International, CC BY 4.0)}

This article is published under the terms of the Creative Commons Attribution License 4.0

https://creativecommons.org/licenses/by/4.0/deed.en US 